\title{
A15
}

doi: 10.14232/ syrpharmacognosy.2021.a15

\section{Isolation and anti-HSV2 studies of compounds from Euphorbia deightonii}

\author{
Muhammad Bello Saidu \\ E-mail: bello.saidu@pharm.u-szeged.hu
}

Background: Euphorbiaceae family has 275 genera and 7,500 species, found in tropical and temperate regions [1,2]. Euphorbia deightonii Croizat, native to West Africa is a cactus-like shrub which grows to $6 \mathrm{~m}$ tall. Historically, it served ornamental, medical, and military purposes [3]. Aims: To isolate compounds from E. deightonii and evaluation of anti-HSV activities of selected compounds.

Methods: Dried plant material $(1.2 \mathrm{~kg})$ was extracted with methanol via percolation. Solventsolvent fractionation with chloroform yielded chloroform portion which then undergo open column chromatography on polyamide using a step gradient of methanol-water to yield four fractions; $20 \%, 60 \%, 80 \%$ and $100 \%$ methanol, respectively. Compounds of the $60 \%$ fraction was purified using NP and RP-VLC, NP and RP HPLC, PLC. Structures were established using NMR and HRMS data.

Results: 38 compounds (1-38) were isolated which are 30 diterpenes (i.e. 27 ingol, 2 ent-atisane and 1 stachane types), 3 tritepenes, 2 lignans, 1 phenyl propanoid, 1 coumarin and 1 ellagic acid derivative. The research also yielded 11 new compounds: 9 ingol diterpenes, 1 tritepene and 1 lignan. All 8 non-diterpenoids were evaluated for anti-HSV2 activity with acyclovir as positive control. Two new compounds (33 and 34) and two known compounds ( $\mathbf{3 2}$ and $\mathbf{3 7}$ ) showed activity with $\mathrm{IC}_{50}$ of $7.05,11.73,2.42, \mu \mathrm{M}$, and $32.09 \mathrm{nM}$, respectively. Interestingly, coumarin 37 has comparable activity to acyclovir.

Supervisor: Dóra Rédei

\section{Acknowledgements:}

Support from the Development and Innovation Operative Programme (GINOP-2.3.2-15-2016-00012) and Stipendium Hungaricum Scholarship Programme is gratefully acknowledged.

\section{References}

[1] Britannica, Encyclopædia. 2017. “Euphorbiaceae.” Britannica. https://www.britannica.com/topic/list-of-plants-in-thefamily-Euphorbiaceae-2039145

[2] The Plant List. 2013. "Euphorbiaceae." The Plant List. (http://www.theplantlist.org/1.1/browse/A/Euphorbiaceae/).

[3] Burkill HM (1985) Useful Plants of West Tropical Africa. Royal Botanic Gardens, Kew (K), United Kingdom. 